\title{
The pathological changes in the spinal cord after dural tear with and without autologous fascia repair
}

\author{
Yi Song • Shao Li $\cdot$ Bo Song $\cdot$ Yanli Zhang $\cdot$ \\ Wenting Gao $\cdot$ Ning Li $\cdot$ Kai Fan $\cdot$ Jianmei Ma
}

Received: 27 November 2013/Revised: 17 April 2014/ Accepted: 18 April 2014/Published online: 7 May 2014

(C) The Author(s) 2014. This article is published with open access at Springerlink.com

\begin{abstract}
Purpose Dural tear is one of the common complications of spinal surgery leading to cerebrospinal fluid leakage followed by serial secondary symptoms. However, little is known about pathological changes of the spinal cord after dural tear. In the present study, we aimed to study the pathological changes in the spinal cord after dural tear with and without autologous fascia repair.

Methods Sixty Sprague-Dawley rats were used for dural tear and autologous fascia graft repair models. Three days and 1 week after surgery, the pathological changes in the spinal cord were analyzed by immunohistochemistry, Western blot, enzyme-linked immunosorbent assay and spinal somatosensory evoked potentials test.

Results Neuroinflammation was found in the parenchyma of the spinal cord characterized by gliosis, increased expression of inflammatory factors and infiltration of
\end{abstract}

\section{Y. Song}

Graduate School of Dalian Medical University,

Dalian 116044, Liaoning, China

e-mail: songyi19800913@163.com

Y. Song · Y. Zhang · K. Fan $(\bowtie) \cdot$ J. Ma $(\bowtie)$

Department of Anatomy, Dalian Medical University,

West Section No. 9, South Road, Lushun,

Dalian 116044, Liaoning, China

e-mail: fankaidalian@hotmail.com

J. Ma

e-mail: ma_jianmei@hotmail.com

Y. Zhang

e-mail: zyl2080@126.com

S. Li

Department of Physiology, Dalian Medical University,

Dalian 116044, Liaoning, China

e-mail: lishao89@hotmail.com exogenesis immunocells in the rats without repair, which impaired the sensory conduction function of the spinal cord at the early stage of injury. Repairing with autologous fascia could attenuate neuroinflammation and help to maintain normal sensory conduction function of the spinal cord.

Conclusion Dural tear could cause a series of inflammatory reactions in the spinal cord and further impair its sensory conduction function at the early stage of injury. Repairing with autologous fascia was a necessary and effective way to prevent the neuroinflammation and to maintain the normal function of the spinal cord.

Keywords Neuroinflammation - Dural tear - Spinal cord . Autologous fascia repair - Conduction

\author{
B. Song \\ Department of Pathology, Dalian Medical University, \\ Dalian 116044, Liaoning, China \\ e-mail: yr0806@hotmail.com \\ W. Gao \\ Laboratory Animal Center, Dalian Medical University, \\ Dalian 116044, Liaoning, China \\ e-mail: gwtpkwy@aliyun.com \\ N. Li \\ Department of General Surgery, Wafangdian Central Hospital, \\ Dalian 116300, Liaoning, China \\ e-mail: lining_19850101@ hotmail.com
}




\section{Introduction}

Dural tear and cerebrospinal fluid (CSF) leakage are common, even sometimes unavoidable complications of spinal surgery. The incidence of dural tear and CSF leakage in posterior decompression procedures for patients with thoracic myelopathy caused by ossification of the ligamentum flavum (OLF) is $32 \%$ [1], in percutaneous endoscopic lumbar discectomy is $1.1 \%$ [2], in anterior decompression procedures for cervical ossification of the posterior longitudinal ligament (OPLL) ranges from 4.3 to $32 \%$ [3]. Dural tear and CSF leakage can lead to intracranial hypotension headache, CSF pseudocyst, adhesive arachnoiditis, wound infection, and even cerebrospinal meningitis. Some evidences have demonstrated that the risk of postoperative infection, neural damage, or arachnoiditis can be effectively decreased after dural tear is successfully repaired [4]. Therefore, it is imperative to repair it immediately once dural tear occurs. Furthermore, dural repair could reduce connective tissue scar and cystic cavity, enhance tissue spare and restore function after acute spinal cord laceration injury in rats [5-7].

Clinically, dural tear can be repaired with direct suturing, superficial/muscle fascia or artificial fascia coverage with or without fibrin glue. In a retrospective clinical review, Xinzhi et al. [1] found that muscle/fascia was an effective material to repair small and moderate-sized dural defects. However, as far as we know, there are no reports about the pathological changes of the spinal cord in an animal model of pure dural tear without spinal cord injury (SCI), so the specific impacts of dural tear on structure and function of the spinal cord are still unknown. In addition, the effects of dural repair with autologous fascia on the spinal cord as well as the underlying mechanisms also remain lacking in the literature.

The purpose of the current study was to investigate the pathological changes in the spinal cord at an early stage after dural tear in rats, further to explore the effects of autologous fascia repair on the spinal cord and the underlying mechanisms, and to provide some knowledge for studying more effective clinical treatments to prevent postinjury complications.

\section{Materials and methods}

Animals

All procedures were in accordance with the Dalian Medical University Guidelines for the Care and Use of Laboratory Animals and were approved by Laboratory Animal Care and Use Committee of Dalian Medical University. Sixty
Sprague-Dawley female rats, weighing 200-220 g, were used for surgical procedures. The animals were randomly assigned to four groups: laminectomy alone with intact dura (sham), dural tear, dural tear with superficial fascia repair and dural tear with deep fascia repair. There were 15 animals in each group: six animals were used for immunohistochemical and histological detection; six animals were used for enzyme-linked immunosorbent assay (ELISA) and Western blot analysis; three animals were used for spinal somatosensory evoked potentials (SSEPs). All surgical procedures were performed under pentobarbital sodium anesthesia, and all efforts were made to minimize suffering.

\section{Surgical procedures}

Animals were anesthetized with pentobarbital sodium $(30 \mathrm{mg} / \mathrm{kg})$. Following a $1-\mathrm{cm}$ midline skin incision starting from the T10 level, the thoracic fascia was opened bilaterally from midline and bilateral subperiosteal dissection of paravertebral muscles was carried out. A T10 laminectomy was performed on all rats to expose the underlying thoracic spinal cord segments. Except the sham group, a sterile 30 gauge needle was used to puncture a small hole in the dura/arachnoid mater; then using a pair of microscissors, a 3-mm transverse incision of the dural/ arachnoid layer was made, followed by 1-mm longitudinal incisions in each of the dural leaflets [7]. In the repaired groups, just before dural tear was done, an approximately $4 \mathrm{~mm}$ wide and $4 \mathrm{~mm}$ long superficial/deep fascia was dissected free from subcutaneous tissue/erector spinae. The flattened fascia was placed on the dura-defected site. The wounds were closed layer by layer in all groups. After the operation, animals were kept warm until they were awake. All surgical procedures were under sterile conditions. None of the animals received antibiotic therapy.

\section{Tissue preparation}

Up to date, almost all of the time frames about pathological changes in the spinal cord were reported in studies of SCI, but not in that of pure dural tear condition. In SCI, leukocyte infiltration, microglia and macrophages accumulation occurred 1-3 days and/or reached the peak 1 week after injury [8-12]. Referring to these factors, we selected 3 days and 1 week after surgery as the time points to investigate the pathological changes in the spinal cord after dural tear. Three days and 1 week after surgery, rats were anesthetized with pentobarbital sodium $(100 \mathrm{mg} / \mathrm{kg})$ and perfused with phosphate-buffered saline (PBS) followed by $4 \%$ paraformaldehyde solution. The $1 \mathrm{~cm}$-length spinal cords containing the surgical sites were carefully dissected and postfixed with the same fixative at $4{ }^{\circ} \mathrm{C}$ overnight. The 
spinal cords were then cryoprotected overnight in PBS containing $20 \%$ sucrose, embedded in optimal cutting temperature (OCT) compound (McCormick Scientific, USA), and serial $20 \mu \mathrm{m}$ sagittal sections were made with a cryostat (Leica CM 3050 S, Leica Microsystems AG, Germany) and used for the immunohistochemistry (IHC) and hematoxylin/eosin (HE) staining.

For ELISA and Western blot analysis, rats were anesthetized with pentobarbital sodium $(100 \mathrm{mg} / \mathrm{kg}) 3$ days and 1 week after surgery, respectively. Then, $1 \mathrm{~cm}$-length spinal cords containing the surgical sites were carefully dissected and stored at $-80{ }^{\circ} \mathrm{C}$ for protein extraction.

Immunohistochemical and histological detection

IHC analysis was performed as described previously [13]. The following antibodies were used: rabbit antiGFAP polyclonal antibody (Dako, Denmark), rabbit antiIba1 polyclonal antibody (Wako, Japan), rabbit antiNitric Oxide Synthase II (iNOS) polyclonal antibody (Millipore Corporation, USA), goat anti-IL-1 $\beta$ polyclonal antibody (R\&D Systems, Inc., USA), rat anti-MBP polyclonal antibody (Abcam Ltd, USA). Cryostat sections from each group were routinely stained with $\mathrm{HE}$ for histological observation. After IHC and HE staining, images were captured using the Nikon digital camera system (DS-Fi1) in combination with microscopy (Nikon eclipse 80i).

\section{ELISA}

Spinal cord tissues of rats were prepared as described above. Proteins were extracted using Nucleus and Cytoplasm Protein Extraction Kit (KeyGEN BioTECH, China) according to the manufacturer's instructions. The concentration of the total proteins in each group was firstly determined using the BCA Protein Assay Kit (AMRESCO LLC, USA). Then, rat IL-1 $\beta$ Mini ELISA Development Kit (PeproTech, USA) was used for the quantitative measurement of IL-1 $\beta$ in each group according to the manufacturer's instructions. The concentration ratio of IL-1 $\beta$ to total proteins in each group was finally used for statistical analysis.

\section{Western blot analysis}

Spinal cord tissue preparation and protein extraction were done as described above. Western blot analysis was performed by the method described previously [14]. The primary antibodies used for the analysis included rabbit anti-iNOS polyclonal antibody (Millipore Corporation, US) and mouse anti- $\beta$-actin monoclonal antibody (Santa
Cruz Biotechnology, Inc., USA). Horseradish peroxidaseconjugated antibodies against rabbit or mouse (Santa Cruz Biotechnology) were used as the secondary antibodies. Protein bands were visualized with a chemiluminescence detection system using the Super Signal substrate.

\section{SSEPs test}

Rats were anesthetized with pentobarbital sodium $(30 \mathrm{mg} /$ $\mathrm{kg})$. An incision was made on the head along the midline and a transcranial screw electrode (E363/20; Plastics One Inc.) was implanted on the somatosensory cortex in left hemisphere receiving input from sensory pathways originating in the right hindlimb. On the left hemisphere, the hindlimb recording electrode was implanted $2.5 \mathrm{~mm}$ posterior to bregma and $2.8 \mathrm{~mm}$ lateral to midline [15]. The electrode made very light contact with the dura mater, but did not compress the dura or brain structures. Two subcutaneous silver needle electrodes (Chengdu instrument factory, China) were used to electrically stimulate the sciatic nerve of right hindlimb. Care was taken to avoid direct contact with the nerve bundle by making sure that only the corresponding limb twitched without tremors on any other limb or area of the animal. An isolated constant voltage stimulator (BL-420S Biological signal collection and processing system) was used for the electrical stimulation of the hindlimb and analysis of data collected. Positive voltage pulses of $3 \mathrm{~V}$ magnitude and 200 microsecond duration at a frequency of $0.5 \mathrm{~Hz}$ were used for hindlimb stimulation. Cortical SSEPs from the transcranial electrodes were amplified by BL-420S with a gain of $500 \mu \mathrm{V}$. The signal was transferred to a personal computer via an optical data acquisition system with one input channel at a sampling rate of 5,000 Hz. Somatosensoryevoked potentials were recorded from anesthetized rats, and the level of anesthesia was kept uniform throughout the recording sessions. Prior to surgery, baseline SSEPs signals were recorded for approximately 100 sweeps. Post-injury SSEPs were recorded after $30 \mathrm{~min}, 3$ days and 1 week for about 100 sweeps to ensure signal stability. The signal to noise ratio was improved by moving averaging of 100 stimulus-locked sweeps.

Statistical analysis

Data were expressed as mean \pm standard error of the mean (SEM) from three independent experiments. All statistical analyses were performed using the Statistical Package for Social Sciences (Version 11.5). The data were evaluated for statistical significance with paired $t$ test. $P<0.05$ was considered statistically significant. 
Fig. 1 Activation of microglial cells in the spinal cord following dural tear Iba-1 IHC staining was performed in the spinal cord in sham group (a and e), unrepaired group (b and f), superficial fascia repair group (c and $\mathbf{g}$ ), and deep fascia repair group (d and h) 3 days after surgery (ad) and 1 week $(\mathbf{e}-\mathbf{h})$, respectively. A representative microglial cell in each group was enlarged and shown in the small quadrilateral, respectively. Quantitative analysis of microglial cells 3 days and 1 week after surgery is shown in $\mathbf{i}$. $* P<0.05, n=3$ per group. Scale bar $100 \mu \mathrm{m}$ in $\mathbf{a}$ to $\mathbf{h} ; 5 \mu \mathrm{m}$ in the small quadrilaterals in $\mathbf{a}-\mathbf{h}$
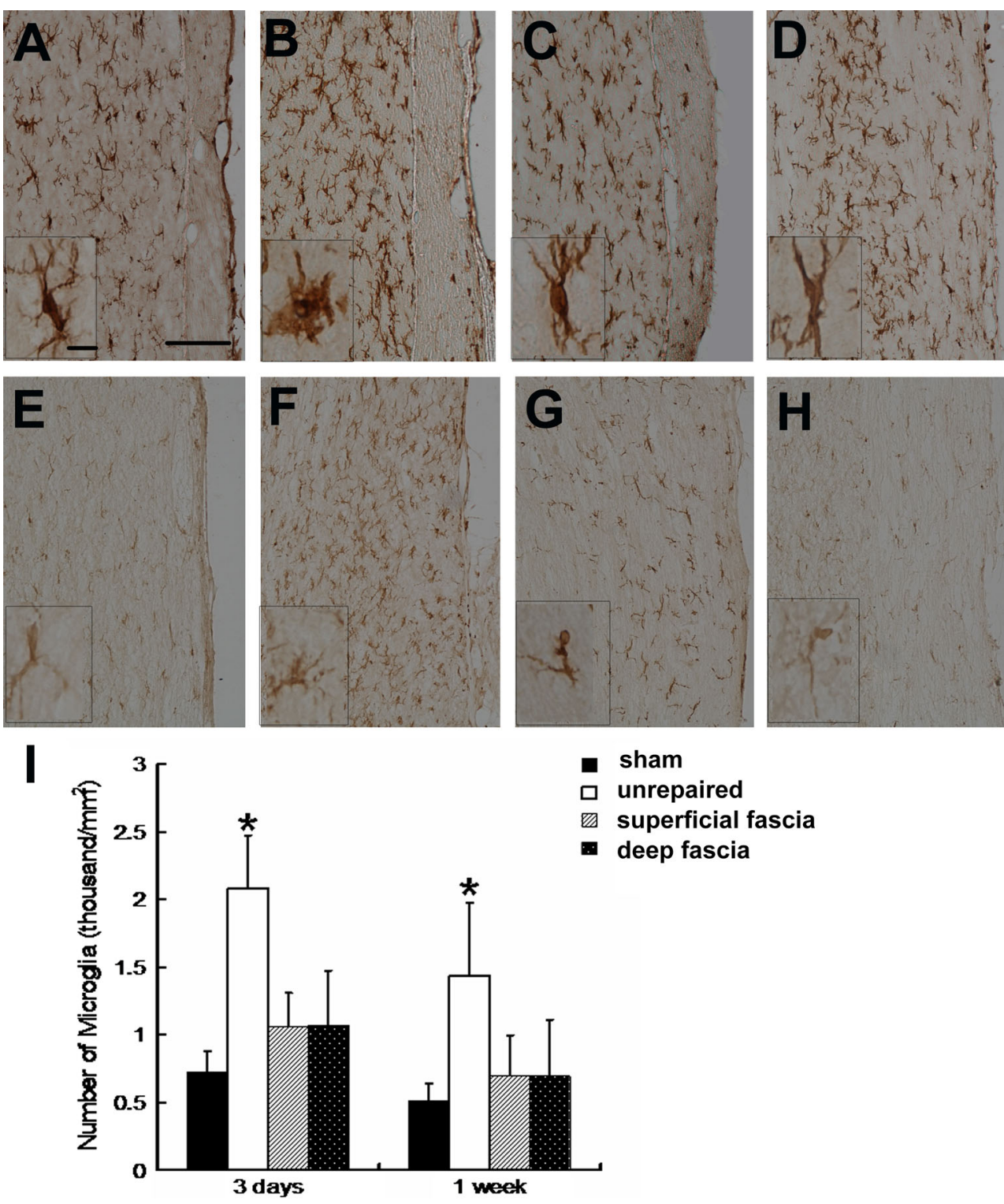

\section{Results}

Dural tear causes inflammatory reactions in the epidural space and gliosis in the spinal cord, which can be alleviated by autologous fascia graft repair

First, we checked the epidural space. Three days after injury, we found some effusion (might be leaked CSF and blood) gathering in the epidural space of the unrepaired group, then the effusion mostly turned into granulation tissue 1 week after injury. Contrarily, no effusion was found in the sham or the superficial/deep fascia repair groups. Next, we studied the status of the glial activation which was one of the prominent characteristics for the neuroinflammation in the central nervous system (CNS) $[16,17]$. The IHC staining (Fig. 1a-d) using Iba-1 (ionized calcium binding adaptor molecule-1) as a specific marker for microglial cells and monocytes showed that 3 days after surgery, Iba-1 positive cells in all groups displayed an activated manner with thicker and shorter processes as well as markedly enlarged cell bodies (small quadrilateral in Fig. 1a-d). Some microglial cells, especially in the unrepaired group (small quadrilateral in Fig. 1b), showed an ameboid shape indicating severe activation. One week after surgery, almost all the microglial cells in the sham and the superficial/deep fascia repair groups (small quadrilateral in Fig. 1e, g and $\mathrm{h}$ ) returned into an unactivated and ramified status except that in the unrepaired group (small quadrilateral in Fig. 1f). Also, we quantified the number of Iba-1 positive microglial cells in the spinal cord 3 days and 1 week after surgery, respectively (Fig. 1i). The results showed that after 3 days, the number of activated 
Fig. 2 Activation of astrocytes in the spinal cord following dural tear GFAP IHC staining was performed in the spinal cord in sham group (a and e), unrepaired group (b and $\mathbf{f}$ ), superficial fascia repair group (c and $\mathbf{g}$ ), and deep fascia repair group (d and h) after surgery for 3 days (a-d) and 1 week $(\mathbf{e}-\mathbf{h})$, respectively. The mean gray values for GFAP-positive signals on 3 days and 1 week after surgery were quantified and shown in i. $* P<0.05$, $n=3$ per group. Scale bar $100 \mu \mathrm{m}$ in $\mathbf{a}-\mathbf{h}$
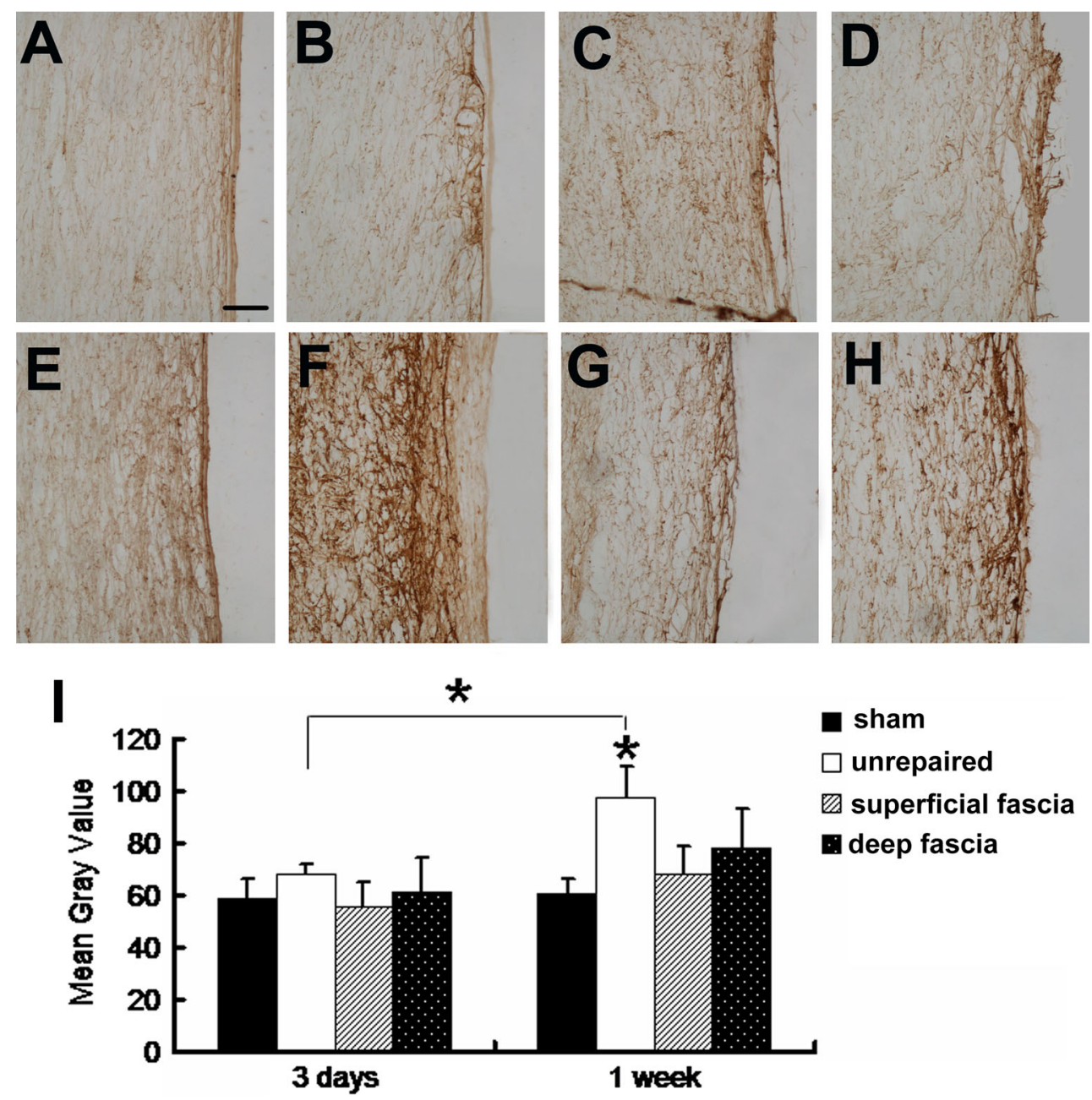

sham $\square$ unrepaired superficial fascia deep fascia microglial cells in the unrepaired group was significantly higher than that in the sham and the superficial/deep fascia repair groups (left columns in Fig. 1i). After 1 week, the number of activated microglial cells in each group relatively went down compared with that of 3 days after surgery. However, in the unrepaired group, it persisted at a significantly higher level, compared with that in the sham and the superficial/deep fascia repair groups (Fig. 1i right columns).

Also, the status of astrocytes was detected by IHC staining using GFAP (glial fibrillary acidic protein) as the marker (Fig. 2a-h). We found that there were no significant differences in GFAP-immunoreactivity among groups (Fig. 2a-d) 3 days after surgery. But 1 week after surgery, stronger GFAP signals and increased GFAP-positive astrocytes with more hypertrophic processes were observed in the unrepaired group (Fig. 2f), compared with that in the sham group (Fig. 2e) as well as that in the superficial/deep fascia repair groups (Fig. $2 \mathrm{~g}$, h). For quantitative analysis by mean gray value detection (left columns in Fig. 2i), no significant differences were found among groups 3 days after surgery. However, 1 week after surgery (right columns in Fig. 2i), the level of mean gray value of each group showed relatively higher than that in 3 days after surgery. Furthermore, the mean gray value of the unrepaired group was significantly higher than that of the sham and the repaired groups.

Repairing with autologous fascia can decrease the expression of inflammatory factors as well as the infiltration of blood-borne inflammatory cells in the spinal cord after dural tear

Because expression of some inflammatory factors and infiltration of blood-borne inflammatory cells may be responsible for glial activation [18, 19], next we detected the expression of inflammatory factors in the parenchyma of the spinal cord after dural tear with and without fascia repair. Pro-inflammatory factor IL-1 $\beta$ IHC analysis was firstly performed. Three days after surgery, stronger IL-1 $\beta$ immunopositive signals with microglial morphology were only detected in the unrepaired group (small quadrilateral 
Fig. 3 The expression of proinflammatory factor IL- $1 \beta$ in the spinal cord following dural tear IL-1 $\beta$ IHC staining was performed in the spinal cord in sham group (a and $\mathbf{e}$ ), unrepaired group (b and $\mathbf{f}$ ), superficial fascia repair group (c and $\mathbf{g}$ ), and deep fascia repair group ( $\mathbf{d}$ and $\mathbf{h}$ ) after surgery for 3 days $(\mathbf{a}-\mathbf{d})$ and 1 week $(\mathbf{e}-\mathbf{h})$, respectively. The small quadrilateral in b shows IL-1 $\beta$ positive microglia-like cells.

The IL-1 $\beta$-positive cells in the area of the right upper quadrilateral were enlarged in the left lower one in $\mathbf{f}$. The HE staining was performed in the serial section of $\mathbf{f}$, and the area equal to the right upper quadrilateral area in $\mathbf{f}$ was enlarged and shown in i. In $\mathbf{i}$, black arrowheads show macrophage-shaped cells, white arrowhead shows lymphocytes and dash arrowhead shows neutrophils, respectively. Quantitative analysis of the IL$1 \beta$ expression by ELISA is shown in $\mathbf{j}$. ${ }^{*} P<0.05, n=3$ per group. Scale bar $100 \mu \mathrm{m}$ in $\mathbf{a}-\mathbf{h} ; 5 \mu \mathrm{m}$ in the small quadrilaterals in $\mathbf{b}$ and $\mathbf{f} ; 5 \mu \mathrm{m}$ in $\mathbf{i}$
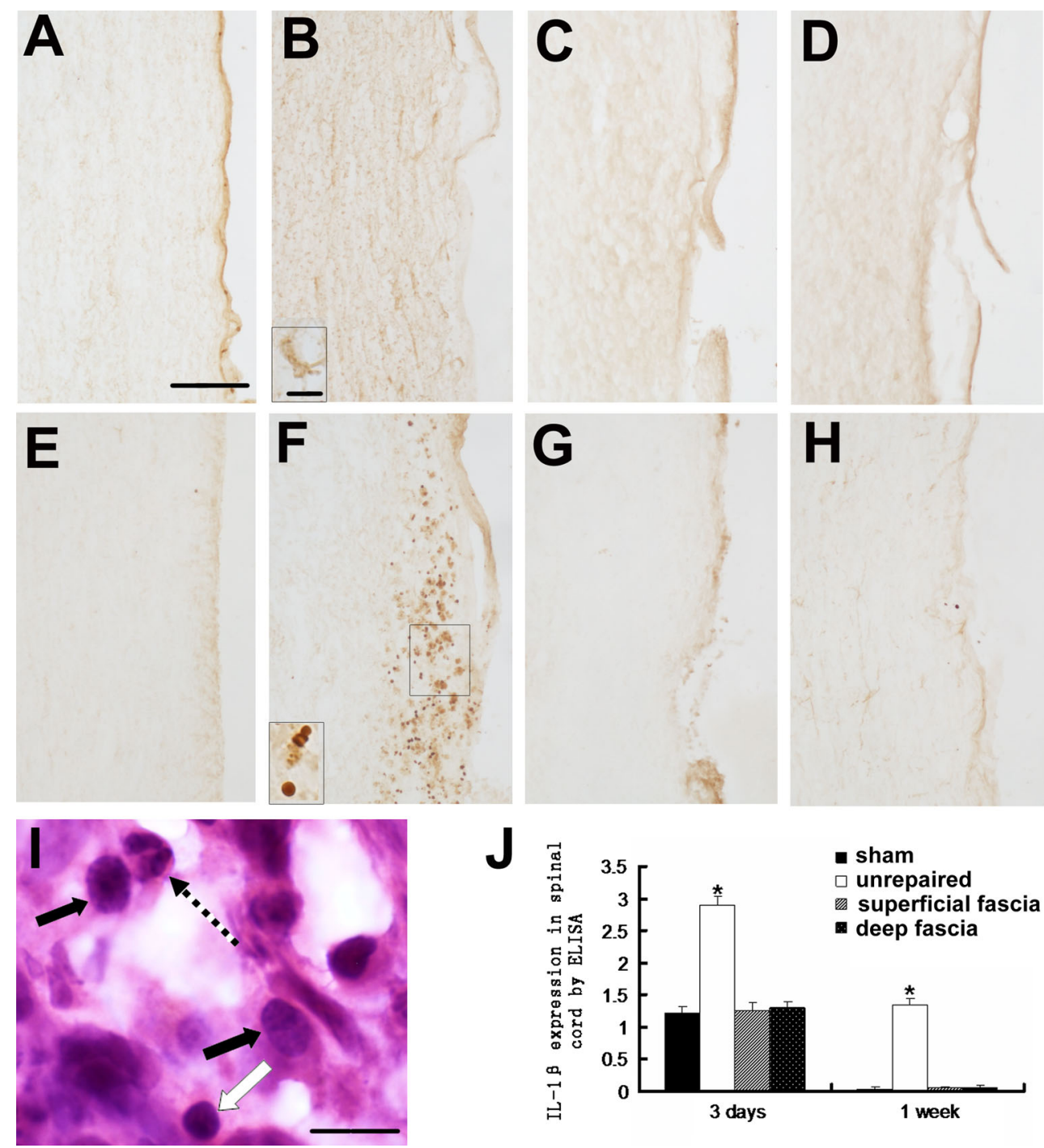

in Fig. 3b), but not in the sham and the repaired groups (Fig. 3a, c and d). One week after surgery, a mass of IL-1 $\beta$ positive cells was found in the parenchyma of the spinal cord in the unrepaired group (Fig. 3f), but not in the sham and the repaired groups (Fig. 3e, g and h). As shown by the morphology, some of the IL-1 $\beta$-positive cells seemed to be infiltrated inflammatory cells with round cell bodies (small quadrilateral in Fig. 3f). To identify cell types of these infiltrated inflammatory cells, HE staining was further performed in serial sections. The results showed that most of the infiltrated inflammatory cells were macrophages (black arrowheads in Fig. 3i), and a few were lymphocytes (white arrowhead in Fig. 3i) and neutrophils (dash arrowhead in Fig. 3i). Then, we examined the IL-1 $\beta$ expression level in each group 3 days and 1 week after surgery by ELISA (Fig. 3j). The results showed that both 3 days and 1 week after surgery in the unrepaired group, the expression of IL-1 $\beta$ was significantly higher than that in the sham and the repaired groups.
The expression of iNOS (inducible nitric oxide synthase) which is another important inflammatory factor that can be induced by IL-1 $\beta$ [20-22] was further investigated in the parenchyma of the spinal cord by IHC staining (Fig. $4 \mathrm{a}-\mathrm{h}$ ). Both 3 days and 1 week after surgery, significantly stronger iNOS-immunopositive signals were detected in the unrepaired group (Fig. $4 \mathrm{~b}$ and f), and no significant differences were found between the sham and the repaired groups. Also, in the unrepaired group, some iNOS-expressing blood-borne-inflammatory-cell-like cells were observed in the spinal cord parenchyma near the dura defect site 1 week after the injury (Fig. 4f). Similar iNOSpositive cells were occasionally found on the surface of the spinal cord in the sham group (arrowhead in Fig. 4e). They did not infiltrate into the spinal cord parenchyma due to the integrity of spinal dura. Then, we examined the iNOS expression levels in each group 3 days and 1 week after surgery by Western blot analysis (Fig. 4i). Both 3 days and 1 week after surgery, the iNOS protein expression was 
Fig. 4 The expression of inflammatory factor iNOS in the spinal cord following dural tear IHC staining was performed in the spinal cord in sham group (a and e), unrepaired group (b and f), superficial fascia repair group (c and $\mathbf{g}$ ), and deep fascia repair group (d and h) after surgery for 3 days (ad) and 1 week $(\mathbf{e}-\mathbf{h})$,

respectively. The black arrow in e shows inflammatory tissue outside the spinal cord parenchyma, and black line in e shows outline of the intact dura. Western blot images of iNOS expression in the spinal cord after surgery for 3 days and 1 week are shown in i. Quantitative analysis of Western blot for the iNOS expression is shown in $\mathbf{j}$. $* P<0.05, n=3$ per group. Scale bar $100 \mu \mathrm{m}$ in $\mathbf{a}-\mathbf{h}$
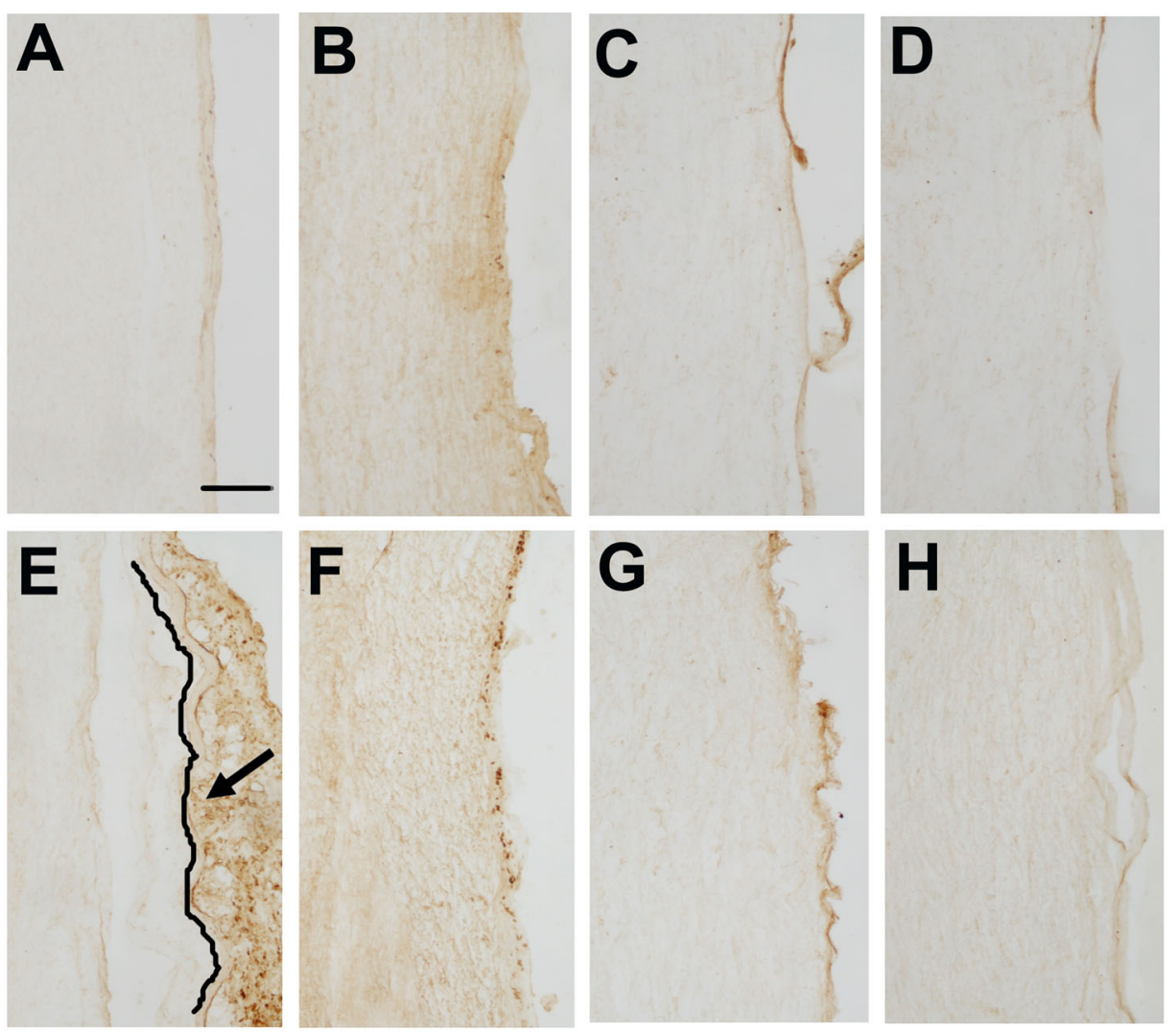

\section{3 days}

1 week
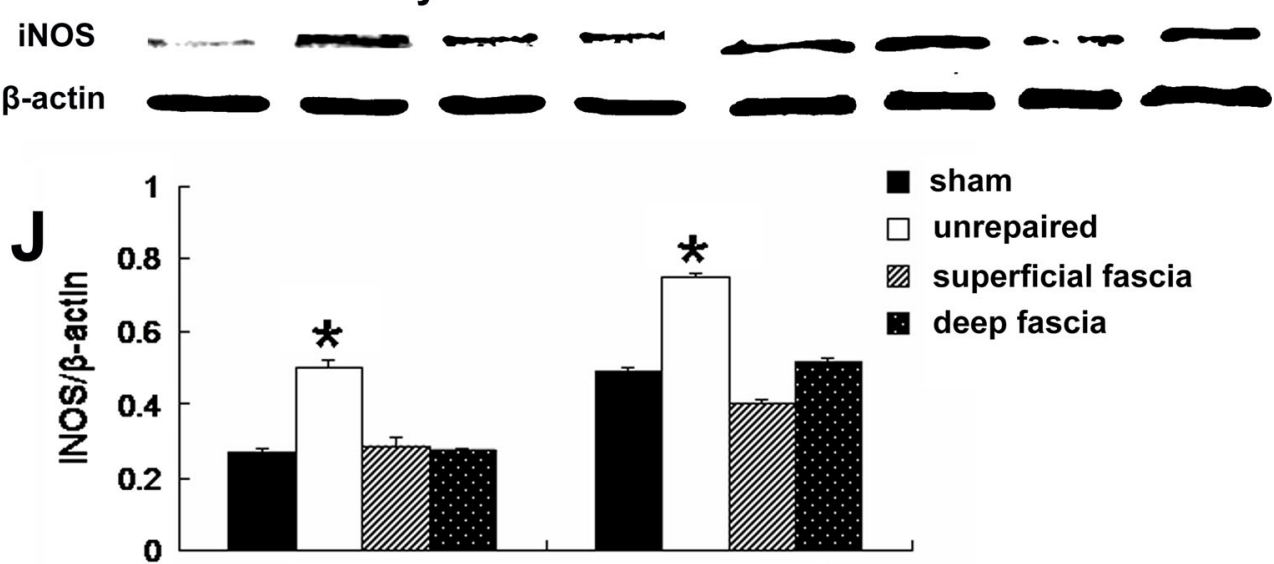

3 days sham

$\square$ unrepaired

\uperficial fascia

deep fascia significantly higher in the unrepaired group, compared with that in the sham and the repaired groups (Fig. 4j).

Repairing with autologous fascia can avoid the impairment of the spinal sensory conduction function caused by inflammation

It has been well known that the inflammatory events in the acute phase of SCI can exacerbate the initial trauma to the spinal cord leading to poor functional recovery [23, 24].
However, whether the neuroinflammatory events caused by dural tear without initial trauma in the parenchyma of the spinal cord could impact on the spinal cord conduction function remains unknown. Thus, we performed SSEPs trial to test the conduction function of dorsal funiculus of spinal cord. The SSEPs recorded from electrodes showed a consistent, reproducible and high negative peak wave preceded by a positive wave. Latencies were measured to the negative wave peak for the N1 waves (Fig. 5 Aa) from pre-injury, $30 \mathrm{~min}, 3$ days, and 1 week post-injury, 


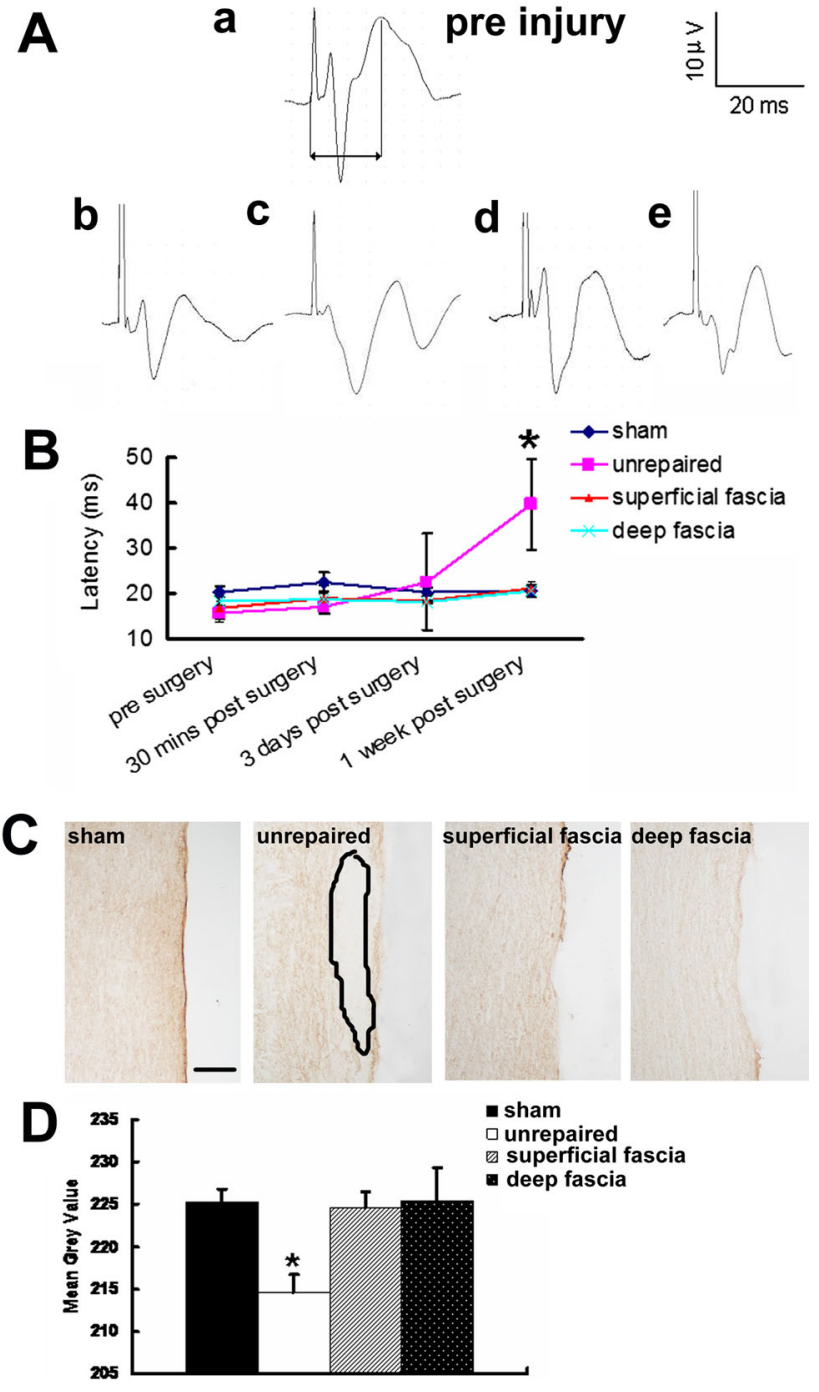

Fig. 5 Latency changes of SSEPs following dural tear A Representative traces of SSEPs from each group, $a$ pre-surgery group, the double arrow line shows the measuring method for the latency; $b-e$ SSEPs waveform of 1-week post-surgery time from sham group $(b)$, unrepaired group $(c)$, superficial fascia repair group $(d)$ and deep fascia repair group $(e)$. B Quantitative analysis of the latency between pre-surgery and certain post-surgery time point in each group. Significant elongation of latency compared with pre-injury was only found in unrepaired group 1-week post-injury. $* P<0.05, n=3$ per group. C The MBP IHC staining of the spinal cord in each group 1 week after dural tear. The outlined area in unrepaired group showed a significant MBP signal lacking area. D The mean gray values for MBP positive signals 1 week after surgery were quantified and the unrepaired group showed significantly lower level. ${ }^{*} P<0.05, n=3$ per group. Scale bar $100 \mu \mathrm{m}(\mathbf{C})$

respectively (Fig. 5A). Significant elongation of latency compared with pre-injury $(15.7 \pm 1.5 \mathrm{~ms})$ was only observed in the unrepaired group 1 week post-injury $(39.8 \pm 10.0 \mathrm{~ms})(P<0.05)$. There were no significant changes for the latency in the sham $(20.4 \pm 1.3 \mathrm{~ms}$ vs $20.5 \pm 1.2 \mathrm{~ms}$ ) and the superficial/deep fascia repair groups $(16.8 \pm 1.7 \mathrm{~ms}$ vs $21.1 \pm 1.3 \mathrm{~ms} / 18.5 \pm 3.0 \mathrm{~ms}$ vs $20.5 \pm 1.4 \mathrm{~ms}$ ) (Fig. 5B). These results indicated that the neuroinflammatory events in the spinal cord caused by dural tear could impact on conduction of the spinal cord. To unveil the underlying mechanisms, we detected the myelin status of the spinal cord 1 week after surgery by MBP (myelin basic protein) IHC staining. Only in the unrepaired group, demyelinated regions shown by significant MBP signals losing (surrounded by dash line in Fig. 5C) were found in the white matter of dorsal funiculus near the dural tear site. For quantitative analysis of mean gray value (Fig. 5D), the MBP signals in the unrepaired group were significantly weaker than those in the sham and the repaired groups.

\section{Discussion}

When the spinal cord is damaged, it undergoes a variety of inflammatory and/or immune responses including the gliosis, infiltration of leukocytes into the injured area, and increased concentration of inflammatory factors [25-27]. In our study, we reported that dural tear could also lead to these pathological changes in the parenchyma of the spinal cord at the early stage of injury. Three days after injury in response to dural tear, the resident microglial cells of the spinal cord were significantly activated and some became macrophage-like cells. Concomitantly, inflammatory factors, IL-1 $\beta$ and iNOS, were increased significantly, especially IL- $1 \beta$ mainly produced by cells with microglial morphology. In contrast, the astrocytes were not significantly activated. Therefore, 3 days after dural tear, the inflammatory reactions in the spinal cord may be caused by mechanical stimuli from dural tear and CSF leakage. And these inflammatory reactions were dominated by activated microglial cells acting as the first form of active immune defense in the CNS. One week after injury, besides microglial cells activation, significantly activated astrocytes and robust infiltrated immunocells were found in the spinal cord near the dural defect site. Meanwhile, the expression levels of inflammatory factors became higher than those in 3 days after injury or persisted at a relatively high level. Thus, 1 week after dural tear, absence of the barrier of dura mater allowed inflammatory reactions in the epidural space to spread into the spinal cord parenchyma and resulted in more severe neuroinflammation. It is well known that inflammation can directly attack the myelin sheath or damage the oligodendrocytes that produce and maintain myelin sheath, resulting in myelin injury and slow nerve impulses conduction $[28,29]$. In the present study, myelin sheath injury was found in the dural tear rat model, and functional elongation of SSEPs latency was also provided as the proof. These indicate that neuroinflammatory 
reactions caused by dural tear could impair the myelin sheath, and then affect conduction of the spinal cord. However, superficial/deep fascia repair could prevent the occurrence of this consequence.

Autologous fascia does not transmit disease nor has the problems of tissue incompatibility and immunological rejection, and they are also costless, so they are popular substitutes for repairing dural tear. Our results indicate that repairing with autologous fascia can pad the epidural space and patch the dura defect site to prevent the CSF leakage, which may improve the pro-inflammatory environment and reduce the inflammatory reactions around the spinal cord. Furthermore, autologous fascia as the barrier between the spinal cord parenchyma and the surrounding tissues can alleviate gliosis of microglial cells/astrocytes caused by mechanical stimuli or inflammatory factors expression, and prevent the infiltration of exogenesis inflammatory cells so as to maintain the normal spinal sensory conduction function. In our study, neither the superficial fascia nor the deep fascia had priority over each other. Therefore, it is up to the clinical surgeons' experience to decide which one to use for dural repair.

Acknowledgments This work has been supported by the National Natural Science Foundation of China (Grant Numbers 81271322) and the Research Fund for the Doctoral Program of Higher Education of China (No. 20122105110002) to JM.

\section{Conflict of interest None.}

Open Access This article is distributed under the terms of the Creative Commons Attribution License which permits any use, distribution, and reproduction in any medium, provided the original author(s) and the source are credited.

\section{References}

1. Sun X, Sun C, Liu X, Liu Z, Qi Q, Guo Z, Leng H, Chen Z (2012) The frequency and treatment of dural tear and cerebrospinal fluid leakage in 266 patients with thoracic myelopathy caused by ossification of the ligamentum flavum. Spine 37:E702-E707

2. Ahn Y, Lee HY, Lee SH, Lee JH (2011) Dural tear in percutaneous endoscopic lumbar discectomy. Eur Spine J 20:58-64

3. Mazur M, Jost GF, Schmidt MH, Bisson EF (2011) Management of cerebrospinal fluid leaks after anterior decompression for ossification of the posterior longitudinal ligament: a review of the literature. Neurosurg Focus 30:E13

4. Wang JC, Bohlman HH, Riew KD (1998) Dural tear secondary to operations on the lumbar spine. Management and results after a two-year-minimum follow-up of eighty-eight patients. J Bone Jt Surg Am 80:1728-1732

5. Liang H, Li C, Gao A, Liang P, Shao Y, Lin T, Zhang X (2012) Spinal duraplasty with two novel substitutes restored locomotor function after acute laceration spinal cord injury in rats. J Biomed Mater Res B Appl Biomater 100:2131-2140

6. Zhang YP, Iannotti C, Shields LB, Han Y, Burke DA, Xu XM, Shields CB (2004) Dural closure, cord approximation, and clot removal: enhancement of tissue sparing in a novel laceration spinal cord injury model. J Neurosurg 100:343-352
7. Iannotti C, Zhang YP, Shields LB, Han Y, Burke DA, Xu XM, Shields CB (2006) Dural repair reduces connective tissue scar invasion and cystic cavity formation after acute spinal cord laceration injury in adult rats. J Neurotrauma 23:853-865

8. Blight AR (1992) Macrophages and inflammatory damage in spinal cord injury. J Neurotrauma 1:S83-S91

9. Carlson SL, Parrish ME, Springer JE, Doty K, Dossett L (1998) Acute inflammatory response in spinal cord following impact injury. Exp Neurol 151:77-88

10. Fleming JC, Norenberg MD, Ramsay DA, Dekaban GA, Marcillo $\mathrm{AE}$, Saenz AD et al (2006) The cellular inflammatory response in human spinal cords after injury. Brain 129:3249-3269

11. Kigerl KA, McGaughy VM, Popovich PG (2006) Comparative analysis of lesion development and intraspinal inflammation in four strains of mice following spinal contusion injury. J Comp Neurol 494:578-594

12. Mueller CA, Schluesener HJ, Conrad S, Pietsch T, Schwab JM (2006) Spinal cord injury-induced expression of the immuneregulatory chemokine interleukin-16 caused by activated microglia/macrophages and CD8 + cells. J Neurosurg Spine 4:233-240

13. Fan K, Wu X, Fan B, Li N, Lin Y, Yao Y, Ma J (2012) Upregulation of microglial cathepsin $\mathrm{C}$ expression and activity in lipopolysaccharide-induced neuroinflammation. J Neuroinflammation 9:96

14. Song B, Wang Y, Kudo K, Gavin EJ, Xi Y, Ju J (2008) miR-192 regulates dihydrofolate reductase and cellular proliferation through the p53-microRNA circuit. Clin Cancer Res 14:8080-8086

15. Agrawal G, Kerr C, Thakor NV, All AH (2010) Characterization of graded multicenter animal spinal cord injury study contusion spinal cord injury using somatosensory-evoked potentials. Spine (Phila Pa 1976) 35:1122-1127

16. Suzumura A (2013) Neuron-microglia interaction in neuroinflammation. Curr Protein Pept Sci 14:16-20

17. O'Callaghan JP, Sriram K, Miller DB (2008) Defining "neuroinflammation". Ann N Y Acad Sci 1139:318-330

18. Takeshita Y, Ransohoff RM (2012) Inflammatory cell trafficking across the blood-brain barrier: chemokine regulation and in vitro models. Ransohoff Immunol Rev 248:228-239

19. Dehghani F, Sayan M, Conrad A, Evers J, Ghadban C, Blaheta R, Korf HW, Hailer NP (2010) Inhibition of microglial and astrocytic inflammatory responses by the immunosuppressant mycophenolate mofetil. Neuropathol Appl Neurobiol 36:598-611

20. Panaro MA, Brandonisio O, Acquafredda A, Sisto M, Mitolo V (2003) Evidences for iNOS expression and nitric oxide production in the human macrophages. Curr Drug Targets Immune Endocr Metabol Disord 3:210-221

21. Knott AB, Bossy-Wetzel E (2009) Nitric oxide in health and disease of the nervous system. Antioxid Redox Signal 11:541-554

22. Nikulina MA, Andersen HU, Karlsen AE, Darville MI, Eizirik DL, Mandrup-Poulsen T (2000) Glutathione depletion inhibits IL-1 beta-stimulated nitric oxide production by reducing inducible nitric oxide synthase gene expression. Cytokine 12:1391-1394

23. Norenberg MD, Smith J, Marcillo A (2004) The pathology of human spinal cord injury: defining the problems. J Neurotrauma 21:429-440

24. Profyris C, Cheema SS, Zang D, Azari MF, Boyle K, Petratos S (2004) Degenerative and regenerative mechanisms governing spinal cord injury. Neurobiol Dis 15:415-436

25. Lu WH, Wang CY, Chen PS, Wang JW, Chuang DM, Yang CS, Tzeng SF (2013) Valproic acid attenuates microgliosis in injured spinal cord and purinergic $\mathrm{P} 2 \mathrm{X} 4$ receptor expression in activated microglia. J Neurosci Res 91:694-705 
26. Bhalala OG, Pan L, Sahni V, McGuire TL, Gruner K, Tourtellotte WG, Kessler JA (2012) microRNA-21 regulates astrocytic response following spinal cord injury. J Neurosci 32:17935-17947

27. Chaitanya GV, Kolli M, Babu PP (2009) Granzyme-b mediated cell death in the spinal cord-injured rat model. Neuropathology 29:270-279
28. Basoglu H, Boylu NT, Kose H (2013) Cuprizone-induced demyelination in Wistar rats; electrophysiological and histological assessment. Eur Rev Med Pharmacol Sci 17:2711-2717

29. Nunes AKDS, Rapôso C, Luna RLDA, Cruz-Höfling MAD, Peixoto CA (2012) Sildenafil (Viagra ${ }^{\circledR}$ ) down regulates cytokines and prevents demyelination in a cuprizone-induced MS mouse model. Cytokine 60:540-551 